\title{
A Step towards Real-Time Customer Relationship Management (CRM)
}

\author{
Muhammad Safeer and Asif Muhammad Malik
}

\begin{abstract}
Over the past two decades especially with the start of 21 st century, Business Intelligence (BI) has emerged as driving force for businesses. Traditional BI tools, methodologies and techniques are being used for strategic decisions. However with the emergence of Real-Time BI (RTBI), goals of real-time responses can be realized. Customer Relationship Management (CRM) is considered as an eye for any successful business. In the recent era, demand of Real-Time CRM is in focus. To achieve the objectives of Real-Time CRM, we have studied the case of CRM and developed a model based on RTBI. Later the model is implemented at an Internet Service Provider (ISP). This work highlights the integration of RTBI with CRM and sets new trends for real-time responses in applications like CRM.
\end{abstract}

Index Terms-Business intelligence (BI), real-time BI (RTBI), customer relationship management (CRM), real-time CRM.

\section{INTRODUCTION}

Over the past two decades especially with the start of $21 \mathrm{st}$ century, Business Intelligence (BI) has emerged as driving force for businesses. Number of writers, academicians and researchers defined BI in different manners. Jayanathi Ranjan [1] stated "BI is the conscious, methodical transformation of data from any and all, data sources into new forms to provide information that is business-driven and results-oriented" with the possibility of automating and integrating maximum functionalities as major objectives. BI is also declared as a new frontier for many enterprises [1]. Malhotra [2] described BI as transporting information to centralized repositories in real-time and supporting analytics for exploiting horizontally and vertically within and outside the company.

Real-Time Business Intelligence (RTBI) means having zero latency inside the processes [3] and providing actionable information to knowledge workers in no time. In the web-based environment, driving force for real-time $\mathrm{BI}$ is e-commerce [4]. BI applications are successfully working in almost every domain. Even in early this century financial services, communications and manufacturing industries were the biggest users of BI [4]. On one hand, improvements in critical areas and overall impact in business is declared as success of BI instead of volume of generating more papers in volume and numbers [1]. On the other hand to increase usage of BI, user-centric solutions are suggested [5].

Heart of $\mathrm{BI}$ is making decisions on the basis of data

Manuscript received December 12, 2012; revised February 13, 2013

The authors are with Shaheed Zulfikar Ali Bhutto Institute of Science and Technology (SZABIST), Islamabad, Pakistan (e-mail: safeeronline@hotmail.com,mAsifQadri@hotmail.com) warehousing and online analytical processing [6]. Earlier BI applications were developed on pre-built data warehouses [3] but now with the emergence of RTBI, Real-Time ETL (extract, transform and load) caches are also being used. The ETL feed the data into data warehouse from operational systems [6]. One of the emerging trends of BI is to embed analytical capabilities in back-end applications like CRM [4].

Customer Relationship Management (CRM) is not a new terminology for industries. It refers to customer-focused strategy [7]. CRM is defined [8] as "a comprehensive strategy and process of acquiring, retaining, and partnering with selective customers to create superior value for the company and the customer". Scott [9] defines CRM as " a set of business processes and overall policies designed to capture, retain and provide service to customers'.

CRM involves number of features including customer service, retention and acquisition. Solving complaints in-time and providing satisfaction to customers is very much necessary for controlling customer churn and insolvency. Bergeron [10] highlights advantages of CRM which includes:

- Greater customer satisfaction

- Greater business coherence

- Managing to increase the number of customers

- Improving and extending customer relationships

- Knowing how to segment customers

- Increasing the effectiveness of providing customer service by having complete, homogeneous information

- Sales and marketing information about customer requirements, expectations and perceptions in real time

Chalmeta [7] categorized CRM into four types such as Transactional CRM, Analytical CRM, Strategic CRM and e-CRM. In e-CRM, customer's information is available in all the time and right time through web services.

This paper is organized as follows: background study is provided in the next section. Proposed model is described in section III while implementation is discussed in section IV. Finally conclusion and future work is presented.

\section{BACKGROUND STUDY}

Business Intelligence as an approach to supporting strategy making of ISP service management is discussed by Li et al. [11]. Based on Model-View-Controller, BI model with six phases as Knowledge Identification, Data Collection, Data Preprocessing, Modeling, Analysis \& Evaluation and Dissemination, is drawn. Attribute-Oriented Induction method is used on IP traffic data of subscribers to discover the customer's usage characteristics and likings [11]. To divide the customers into clusters with different usage 
behavior patterns, Self-Organizing Map technique is applied and finally Recency, Frequency and Monetary modeling is used to regulate customer's value of each cluster [11]. In our model, customers from same area are grouped together and customer's usage characteristics are used to analysis usage patterns in real-time manner.

Future RTBI is predicted by Azvine et al. [3] in 2006. RTBI is declared as "turning data into information into action" and stated as delivery, modeling, analysis of information and actions in real-time. In this work, the authors [3] put infrastructure, data integration and data warehouse as on on-demand items. Technology challenges are group into three layers as Analytics, Data Integration and Operational. Whereas for implementation of RTBI, automatic data analysis, decisions optimizations \& what-if analysis and changing process are proposed [3]. The authors [3] implemented few modules of proposed vision for RTBI in the telecom sector. In our model data integration and data warehouse are considered as complimentary elements. Moreover Real-Time ETL is also proposed to achieve RTBI for CRM application.

Nguyen et al. [12] presented Sense \& Response Service (SARESA) architecture for RTBI implementation in mobile sector to detect the frauds in the field. SARESA is a mechanism to constantly capture events from a mixture of source systems and transform those in nearly real-time performance indicators and intelligent business actions [12]. The technique is also used by Schiefer and Seufert [13] for monitoring supply chain process in real-time manner. In our model events are triggered against database operations like insert \& update rows. The WAP gateway is used as messages indicators.

Zhang et al. [14] discussed a case study of log data mining in telecommunication. The main objective of the study [14] was to conduct the analysis to support customer retention and customer acquisition. In author's [14] point-of-view, log data is available for useful analysis but is not being used. Increasing customer's loyalty is also discussed. For log data analysis [14], data marts generation is done as first step; later data mining is carried and finally based on different scenarios BI results are shown through graphs. In our model, real-time information is made available through ETL cache whereas for analysis, data is extracted from data warehouse.

Working on CRM in telecommunications area was also previously done by Wright et al. [15]. Two case studies from European telecom industry are provided to support the work. These case studies are basically BI solutions to support the companies in CRM related issues. After the implementation of $\mathrm{BI}$, the company planned long-term strategies to guaranteed customer-centric industry. In the second case study, another company [15] has also realized importance of $\mathrm{BI}$ application for its customer acquisition and retention. In our model, RTBI is used with e-CRM to make it more interactive and responsive.

Deng et al. [16] have implemented BI solution in Mobile Electronic Commerce (MEC). Derived from e-commerce, the authors presented MEC framework [16], whereas BI technical structure is alienated into Data Warehouse, OLAP, Data Mining and Enterprise Information Portal phases [16]. The MEC model [16] is staged as mobile customer, mobile network, WAP gateway, MEC platform and financial institution. The solution is implemented with four-layer architecture [16] as Data Storage, Model, Control and View layers [16]. In our model, five basic layers are proposed horizontally and control layer as vertically. To achieve the objectives of RTBI, WAP gateway is also proposed to generate automatic text SMSs for support team and for the customers.

\section{Proposed Model for ReAl-Time CRM}

Background studies, discussions with ISP management and our knowledge provided basis for proposing model for RTBI for ISP-CRM.

Proposed model encompasses basic architecture of business intelligence [11],[16]. Data warehouse is made part of proposed model whereas it was put on-demand by [3]. However for achieving RTBI, real-time ETL cache is also proposed as shown in Fig. 1.

The proposed model s layered as follows:-

- Users Layer

- Operational Layer

- Data Storage Layer

- Analytical Layer

- Viewer Layer

- Control Layer

In the proposed model, user layer provides input to the operational layer. The operational layer is consisting of WAP gateway and disparate databases including CRM \& complaints. Data warehouse and Real-Time ETL are placed into data storage layer while analytical layer facilitates analysis and data mining. Finally viewer layer is providing reports, analysis, dashboards and graphs. These layers are sited horizontally whereas control layer, which is responsible for communication and connectivity among different layers, is placed vertically.

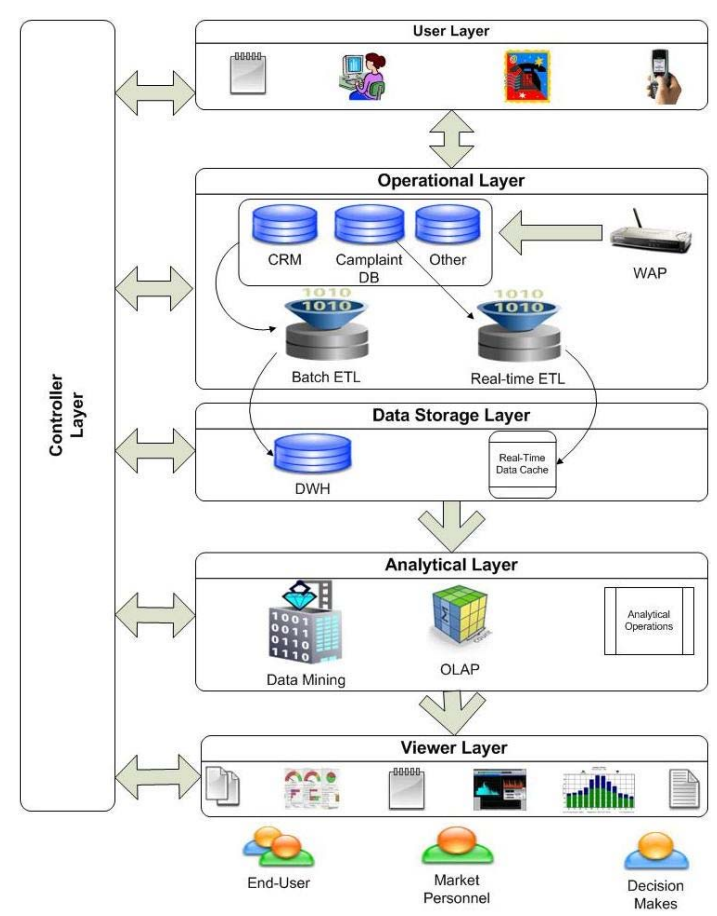

Fig. 1. Proposed model for real-time CRM 
Complaints database residing at operational layer generates triggers for WAP gateway for sending SMS to support team and customers. Whenever a new entry is made into complaints database or a new complaint is logged, triggers are generated on "just-in-time" basis. The complaint can be logged by any of the following ways:-

- via web interface

- via SMS

- via IVR

- via call to help desk

Based on the customer's profile data and circuit's installation information, SMS is generated for support team's filed administrators and/or technicians, deputed at relevant area. The SMS carrys information like token ID, customer address and type of complaint. During and after complaint's resolution, support team provides feedback to system with appropriate flag(s) that reflects real-time status to all stakeholders.

For transferring up-to-date and real-time information from operational layer to upper layers, a real-time ETL cache is proposed at data storage layer. Real-time ETL is completely isolated with data warehouse, placed at the same layer. Contents of data warehouse are updated in batches on periodically basis.

In the analysis layer, analytical and data mining techniques are used for preparing reports for viewer layer while taking inputs from data storage layer. Based on the query, made by the viewer, data is extracted from data warehouse and/or Real-time ETL cache. Finally, in the viewer layer, management is facilitated with analysis, reports, graphs and dashboards. The control layer provides the mean of communication and connectivity among different layers as and when required.

\section{IMPLEMENTATION}

$\mathrm{ABC}$ (we are not disclosing the name because of privacy policy of the company) is one the largest ISP services provider in Pakistan. The company is providing data services across the country. Data services mainly include DSL, intranet, web hosting and video-conferencing. For DSL services, the company has deputed technical administrators and technicians as filed staff for providing up \& running services on $24 \times 7$ basis.

The company has established an emergence and nearly real-time complaint resolution system. Only very few customer support representative (CSRs) are deputed at help desks around the clock, as application is handling events automatically. The role of CSRsX is just to monitor the application and listen complaints on telephone, if received.

A new record is inserted into complaint's application whenever customer logged a complaint, which ultimately triggers some events for field administrators and technicians. While logging new complaint into system, few parameters like customer ID \& type of complaint are required. Other decisions like installed location of circuit, selection of concerned field staff for forwarding of complaints are selected automatically by the system. Complaint ID is sent to customers too for tracking purposes. Customers are also facilitated with web interfaces.

At every update, made by filed administrators and/or technicians, system is updated with new status on "just-in-time" basis. The new updated statuses can be viewed electronically at anytime and in some cases, WAP gateway is involved to update concerned support staff and customers. These new statuses include delays in solving the problem, acquiring new $\mathrm{CPE} /$ modem; help required from other support staff. Finally once the problem is solved, status is updated at all places including sending 'Problem Solved' SMS to the subscriber.

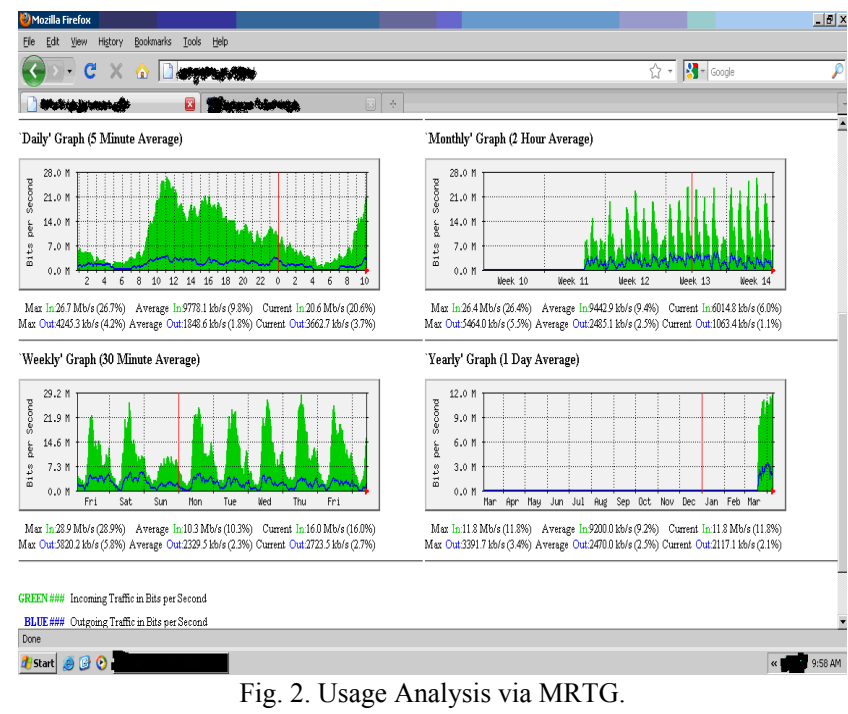

ISP's middle management and CSR can also view status of any complaint, currently in operation along with the history of customer. Current status is queried from operational system while history is extracted from data warehouse. Incase, a complaint resolution is taking too much time, SMSs are also generated for CSRs and middle management as alerts for taking necessary measures.

CSRs and middle management can view customer's history by MRTG, revenue statistics, complaint graphs and analysis reports as shown in Fig. 2 and Fig. 3. Graphs also provide better views about customers, who logged more complaints and areas, from where most complaints are received.

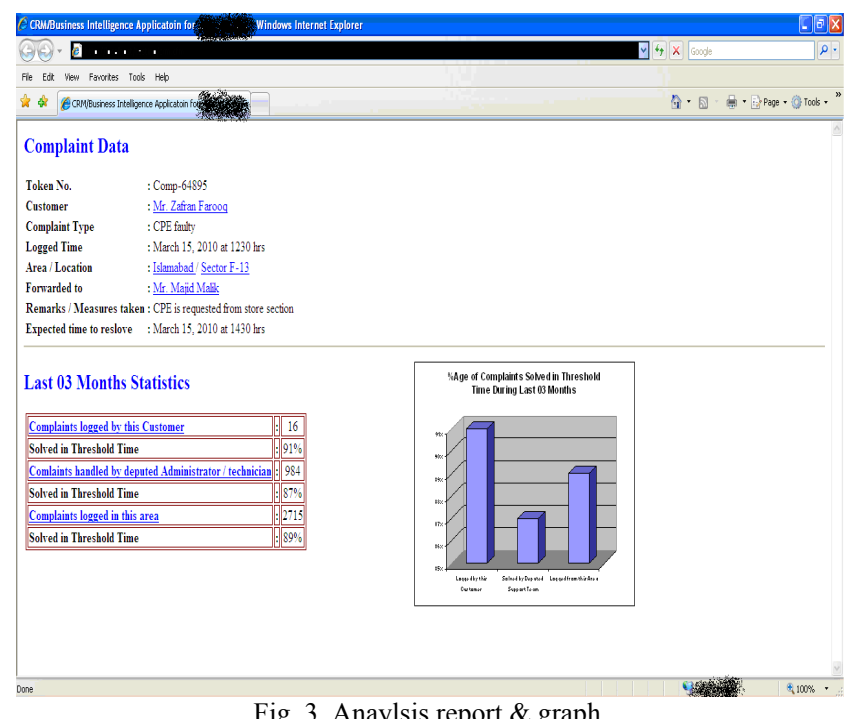

Fig. 3. Anaylsis report \& graph. 
Tools used for systems include Oracle as database, Cold Fusion for web interface of operational application and Oracle Business Intelligence \& Oracle Discoverers for BI analysis. Difference modules are also integrated through custom build Cold Fusion application. The WAP gateway for generating and receiving SMSs is handled by BPA Systems.

\section{CONCLUSION AND FUtURE WORK}

$\mathrm{BI}$ has emerged as driving force for businesses and became a new frontier for many enterprises. After the introduction of real-time BI, its usage is increased. As opposed to traditional BI, RTBI encompasses new trends by allowing the applications in real-time responses. On the other hand, demand of Real-Time CRM is in focus. With the help of which, customer's problems can be addressed in "just-in-time" basis.

In this research work, a five layer model for real-time CRM is proposed based on real-time BI and CRM in which events like complaints logging and resolution are responded in nearly real-time.

In achieving the objectives of Real-Time CRM, analytical reports, dashboards and graphs from proposed RTBI model for CRM application are presented to the users. Finally, empirical validation is provided with the help of a case study in telecom sector. In future, this work can be extended from real-time BI to Pervasive BI.

\section{REFERENCES}

[1] J. Ranjan, "Business Justification with Business Intelligence," Emerald, 2008.

[2] Y. Malhotra, from information management to knowledge management: beyond hi-techhidebound systems, in Srikantaiah, T.K. and Koenig, M.E.D. (Eds), Knowledge Management, Information Today, Inc., Medford, NJ., 2000.

[3] B. Azvine, Z. Cui, D. D. Nauck, and B. Majeed, "Real time business intelligence for the adaptive enterprise," IEEE, 2006.

[4] S. O. Jr., "Is business intelligence a smart move?" Industry Trends, 2002.

[5] M. Psenka, "Planes, trains, and automobiles: The UI in BI," Business Intelligence Journal, vol. 14, no. 1, 2009.

[6] R. T. Herschel and N. E. Jones, "Knowledge management and business intelligence: The importance of integration," Journal of Knowledge Management, Emerlad, 2005.

[7] R. Chalmeta, "Methodology for customer relationship management," The Journal of Systems and Software, vol. 79, no. 7, pp. 1015-1024, 2006.

[8] A. Parvatiyar and J. N. Sheth, "Customer relationship management: emerging practice, process and discipline," Journal of Economic and Social Research, vol. 3, no. 2, pp. 1-34, 2001.

[9] D. Scott, "Understanding organizational evolution: Its impact on management and performance," Quorum Books, 2001.
[10] B. Bergeron, Essentials of CRM: Customer relationship management for executives, John Wiley \& Sons, 2001.

[11] S. T. Li, L. Y. Shue, and S. F. Lee, Business intelligence approach to supporting strategy-making of ISP service management, Elsevier, 2006.

[12] T. M. Nguyen, J. Schiefer, and A. M. Tjoa "Sense \& Response Service Architechture (SARESA): An approach towards a real-time business intelligence solution and its use for a fraud detection application," $A C M, 2005$.

[13] J. Schiefer and A. Seufert "Management and controlling of time-sensitive business processes with sense \& respond," in Proc. Web Technologies and Internet Commerce (CIMCA-IAWTIC'06), vol. 1, 2005.

[14] X. L. Zhang, W. J. Gong, and T. Narita, "Business intelligence in teleommunication enterprises: A case study of log data analysis," IEEE, 2006.

[15] L. T. Wright, M. Stone, and J. Abbott, "The CRM imperative - Practice Vs theory in the telecommunication industry," Journal of Database Marketing, vol. 9, 2002.

[16] X. Deng, C. Jin, and J. Ding, "Process-based requirement anaylsis on business intelligence in mobile electornic commerce," IEEE, 2008.

[17] S. Viaene, "Linking business intelligence into your business," IT Pro/IEEE, November/December 2008.

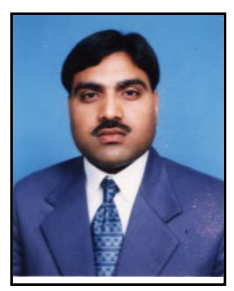

Muhammad Safeer has received his M.S. degree in information technology from Shaheed Zulfikar Ali Bhutto Institute of Science \& Technology (SZABIST), Islamabad, Pakistan in 2010. He is serving as Telecom Billing Professional in one the leading telecom operator in Pakistan for last more than 08 years. Since 2010 , he is also leading billing team there.

Since 2002, he is teaching different IT courses especially related with Databases, Decision Support Systems and Data Warehousing in different institutes/universities as visiting lecturer. Moreover, he has been involved in software development and also supervised number of students in their final projects/thesis. For the last 03 years, he has also been involved in research. So far, there are 06 publications on his record with 03 at IEEE and same number of local publications.

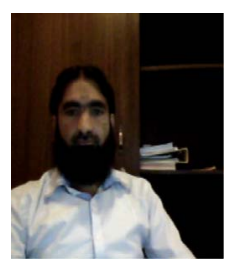

Asif Muhammad Malik has received his B.S. degree in computer sciences from Muhammad Ali Jinnah University, Islamabad in 2003. Later he did M.S. in Software Engineering followed by Professional MBA for Shaheed Zulfikar Ali Bhutto Institute of Science \& Technology (SZABIST), Islamabad, Pakistan.

After graduation in 2003, he joined an I.T. company as a Web Engineer. Microsoft technologies are his main expertise. Till now he has served different organizations and currently working as a Technical Team Lead in one of the leading company in Islamabad, Pakistan. His professional education contains different certifications as well mainly Microsoft Certified Technology Specialist (SharePoint).

So far, he has 03 international research publications. Moreover he was also a part of visiting faculty for 1.5 years at BS level. 